\title{
A Nonlinear Fingerprint-Level Radar Simulation Modeling Method for Specific Emitter Identification
}

\author{
Peng Man ${ }^{1,2,3}$, Chibiao Ding ${ }^{1,3,4, *}$, Wenjuan Ren ${ }^{1,2}$ and Guangluan $\mathrm{Xu}^{1,2}$ \\ 1 Aerospace Information Research Institute, Chinese Academy of Sciences, Beijing 100094, China; \\ manpeng16@mails.ucas.ac.cn (P.M.); wjren2011@mail.ie.ac.cn (W.R.); gluanxu@mail.ie.ac.cn (G.X.) \\ 2 Key Laboratory of Network Information System Technology (NIST), Institute of Electronics, \\ Chinese Academy of Sciences, Beijing 100190, China \\ 3 School of Electronic, Electrical and Communication Engineering, University of Chinese Academy of Sciences, \\ Beijing 100190, China \\ 4 National Key Laboratory of Science and Technology on Microwave Imaging, Beijing 100190, China \\ * Correspondence: cbding@mail.ie.ac.cn
}

Citation: Man, P.; Ding, C.; Ren, W.; $\mathrm{Xu}, \mathrm{G}$. A Nonlinear Fingerprint-Level Radar Simulation Modeling Method for Specific Emitter Identification. Electronics 2021, 10, 1030. https:// doi.org/10.3390/electronics10091030

Academic Editor: Flavio Canavero

Received: 25 March 2021

Accepted: 23 April 2021

Published: 26 April 2021

Publisher's Note: MDPI stays neutral with regard to jurisdictional claims in published maps and institutional affiliations.

Copyright: (c) 2021 by the authors. Licensee MDPI, Basel, Switzerland. This article is an open access article distributed under the terms and conditions of the Creative Commons Attribution (CC BY) license (https:/ / creativecommons.org/licenses/by/ $4.0 /)$.

\begin{abstract}
With the development of information technology for modern military confrontations, radar emitter fingerprint identification has become a hot and difficult topic in the field of electronic warfare, especially in the field of electronic reconnaissance. Owing to the confidentiality of military systems, most of the existing studies use simulation data for radar emitter fingerprint identification experiments and analysis. However, most of the existing modeling methods focus on the mechanism analysis of the nonlinear fingerprint characteristics of a single independent component. Its main disadvantage is that it can only represent the nonlinear fingerprint characteristics of some components in the radar emitter system but cannot fully reflect the nonlinear fingerprint characteristics of the whole radar emitter system. In this paper, a nonlinear fingerprint-level radar simulation modeling method is proposed. In contrast to the previous single component modeling method, the systematic nonlinear characteristic modeling method of this model can provide individual radar signal data under different modulation modes and working parameters, and provide experimental conditions for data support and theoretical analysis of radar emitter fingerprint identification.
\end{abstract}

Keywords: electronic warfare; radar emitter fingerprint identification; fingerprint-level; simulation modeling method

\section{Introduction}

With the rapid development of military technology in the world, weapon systems continue to emerge. However, the basic principle "Know the enemy and know yourself, and you can fight a hundred battles with no danger of defeat" remains unchanged. In the dynamic battlefield environment, how to associate the signal detected from the complex electromagnetic environment with the emitter, the platform and weapon system has important military significance. Hence, the demand for the concept of radar emitter fingerprint identification was generated. Radar emitter fingerprint identification began in the mid-1960s, which is generally called specific emitter identification (SEI) [1-3] in foreign countries. The reason why emitter individual identification is called "specific emitter identification" or "fingerprint identification" is that when radar emitters have the same type and parameters (such as the same batch of radars produced by a certain radar manufacturer, or these radars work in the same signal modulation mode), a specific radar emitter individual can still be uniquely identified. Talbot et al. [1] proposed a typical SEI system structure in 2003, the process is as follows: First, the signal is received by the RF receiving subsystem; after the signal processing, the received signal is preprocessed by filtering, denoising, pulse detection and so on, and the signal is demodulated according to the actual needs. Then, the fingerprint feature is extracted to obtain the fine features 
containing the individual information of the emitter; finally, compared with the database, the specific emitter of the signal is determined by the classification and recognition algorithm, and the individual emitter is identified. In recent years, the theory and practical application of emitter individual identification technology are constantly improved, and the research of fingerprint feature extraction method has made great progress [4-17].

Owing to the confidentiality to military systems, most of the existing studies use simulation data for radar emitter fingerprint identification experiments and analysis. The origin of different individual differences is analyzed from the source by modeling the nonlinear characteristics of radar emitter. However, most of the existing modeling methods focus on the mechanism analysis of the nonlinear fingerprint characteristics of a single independent component [18-22]. Its main disadvantage is that it can only represent the nonlinear fingerprint characteristics of some components in the radar emitter system but cannot fully reflect the nonlinear fingerprint characteristics of the whole radar emitter system. Even if the experimental results show that there are individual differences between the radar emitter signals, it cannot be proved that this difference comes from the above theoretical model. Therefore, the establishment of a nonlinear fingerprint-level radar emitter simulation model is of great significance to the major problems that need to be solved in the field of radar emitter fingerprint identification.

Some works $[18,19]$ build the phase noise model, and construct different individual radar signals by changing the phase noise intensity and phase noise power spectral density distribution, respectively. References $[20,21]$ use the Taylor series model as the nonlinear characteristic model of power amplifier, and different individual radar signals are generated by changing the Taylor series. Thesis [22] uses a polynomial phase model to describe the radar signal source and simulates different individual radar signals by changing phase polynomial coefficients. It can be seen that the above radar signal source simulation schemes are all for the nonlinear simulation of single components such as frequency source or amplifier, and lack of theoretical methods and mathematical models to systematically and comprehensively study the generation mechanism of "fingerprint" features from the overall nonlinear characteristics of radar emitter. However, the quality of the data source is very important in scientific experiments. If the simulation quality of the data source is general, then the conclusion is likely to be wrong. Therefore, it is necessary to build a radar emitter model as close to the real as possible with the help of simulation platform from the overall nonlinear characteristics of radar emitter. In this paper, a nonlinear fingerprintlevel radar simulation modeling method is proposed. In contrast to the previous single component modeling method, the systematic nonlinear characteristic modeling method of this model can provide individual radar signal data under different modulation modes and working parameters and provide experimental conditions for data support and theoretical analysis of radar emitter fingerprint identification. The main contributions are summarized as follows: (1) compared with the traditional independent component modeling method, this model uses systematic modeling method to generate radar emitter signal data; (2) compared with traditional functional simulation modeling methods, this model can provide individual level signal data containing fingerprint features; and (3)compared with the traditional modeling method which can only provide signal data of single working parameter and modulation mode, this model can simulate individual level signal data in a variety of working parameters and modulation modes.

This paper is arranged as follows: Section 2 introduces the structure of a typical radar emitter and the simulation platform. Section 3 analyzes and verifies the nonlinear characteristics of the radar emitter core module including signal source, mixer, and amplifier. Numerous experimental results are shown in this Section. Finally, conclusions are drawn in Section 4. 


\section{Modeling Method}

\subsection{Basic Structure of Radar Transmitter}

The task of the radar transmitter is to provide a high-power transmitting signal for the radar system to meet specific requirements, which is radiated to space by antenna through feeder and transceiver switch. The radar transmitter is mainly divided into two types: single-stage oscillation transmitter and master oscillation power amplification (M.O.P.A.) transmitter. Among them, the composition of the single-stage oscillatory transmitter is relatively simple and the cost is relatively low, but the performance is poor, especially the frequency stability is low, and it does not have phase-coherent characteristic. The composition of the M.O.P.A. transmitter is relatively complex, but it has good performance: it has high frequency stability, can generate complex signal waveform, transmit phase-coherent signal, realize the pulse compression mode and is suitable for broadband frequency agility, etc. [23-25]. So far, most radars, especially instrumentation control radars and phased array radars with high stability and high performance, have adopted the M.O.P.A. transmitter. Therefore, this study mainly discusses the M.O.P.A. transmitter.

The composition of the M.O.P.A. transmitter is shown in Figure 1, which is mainly composed of radio frequency (RF) amplifier chain, pulse modulator, solid-state frequency source and high-voltage power supply. The RF amplifier chain is the core part of the M.O.P.A. transmitter, which is mainly composed of the pre-amplifier, the intermediate RF power amplifier and the output RF power amplifier. Solid-state frequency source is an important part of radar system, as shown in the dotted box in Figure 2. It is mainly composed of high stable reference frequency source, frequency synthesizer, waveform generator and mixer (up-conversion). Solid-state frequency source provides RF transmitting signal frequency, local oscillator (LO) signal frequency, intermediate frequency (IF) coherent oscillation frequency, timing trigger pulse frequency and clock frequency for radar system, the frequency of these signals is controlled by a highly stable reference source, and there is a definite phase relationship between them, which is usually called coherent signals.

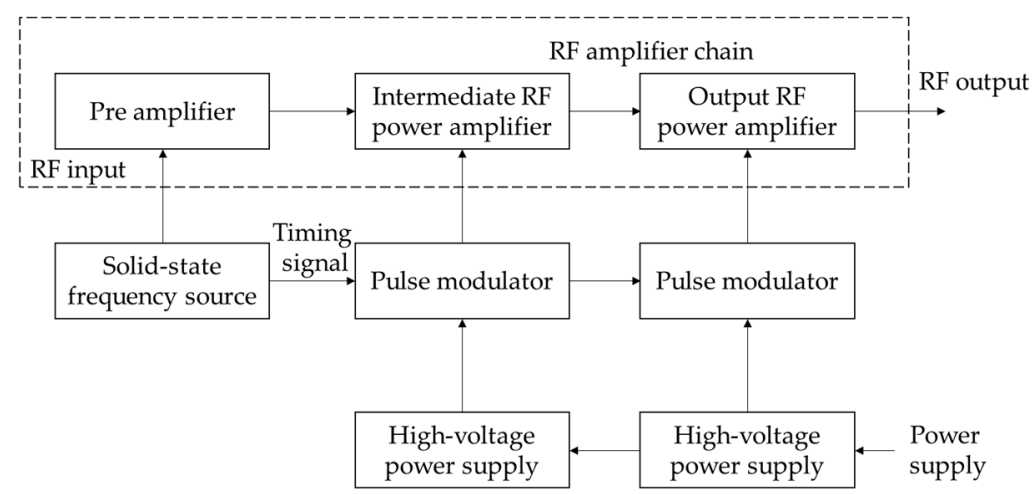

Figure 1. Composition of M.O.P.A. transmitter.

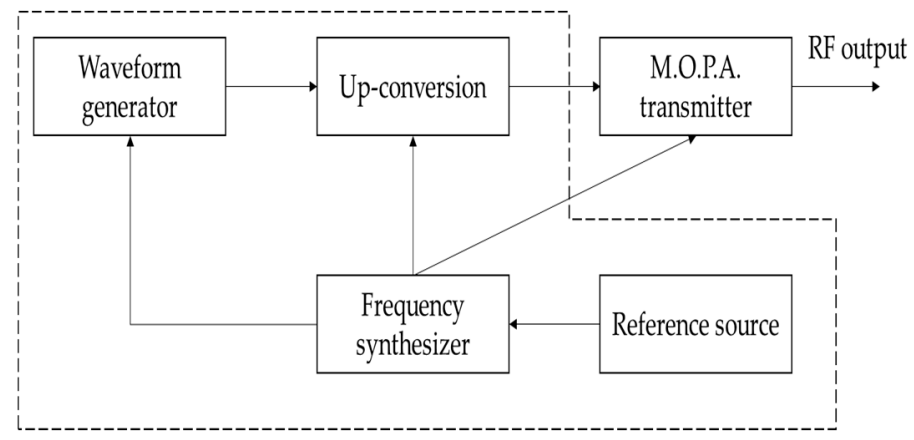

Figure 2. M.O.P.A. transmitter structure of modern coherent radar. 
Based on the characteristics and structure of modern radar emitter transmitter, the structure of radar emitter transmitter is simplified [26], as shown in Figure 3.

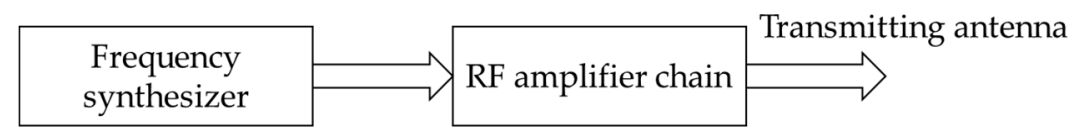

Figure 3. Simplified structure block diagram of radar transmitter.

\subsection{Nonlinear Fingerprint-Level Radar Simulation Modeling}

Systemvue is a system level electronic simulation software of keysight company, which mainly realizes system level modeling and simulation in electronic field, including radar, communication, radio and other fields. This software can realize digital, analog or mixed domain, single rate or multi rate simulation system in the application of digital signal processing, communication equipment, radio and control system. It contains a variety of tool libraries, which is convenient for users to add baseband, RF, physical layer and other functional modules, and supports the theoretical analysis and simulation of analog circuits such as amplifiers, capacitors and inductors; The software interface is friendly, and can be combined with $\mathrm{C}++$, ADS, Xilinx, MATLAB and other software to achieve simulation. In recent years, Systemvue has attracted more and more attention in the field of electronic simulation, especially in the field of communication radar. At present, the use of this software in the electronic field is mainly reflected in the communication system, whereas the construction and simulation of the radar system platform is less. Systemvue is a system level design and simulation software for baseband and RF advanced architecture development. It includes a variety of simulation techniques in time-frequency domain, baseband radio frequency domain, and can realize all linear and nonlinear behavior level modeling and simulation of baseband and radio frequency system. It mainly supports the simulation of the following spectrum types: (1) spectrum of signal source and carrier frequency; (2) intermodulation/harmonics: nonlinearity caused by RF devices such as mixers, amplifiers, etc.; (3) broadband noise: caused by thermal noise of RF circuit; (4) phase noise: phase noise transmitted in RF system; These functions make it very suitable for nonlinear fingerprint-level radar simulation modeling.

According to the working principle of the radar, the radar system is designed and modeled based on the Systemvue simulation platform, as shown in the Figure 4 . The upper part of the model is the signal source, and the lower part is the IF filter, IF amplifier, mixer, local oscillator, RF filter and RF amplifier from left to right.

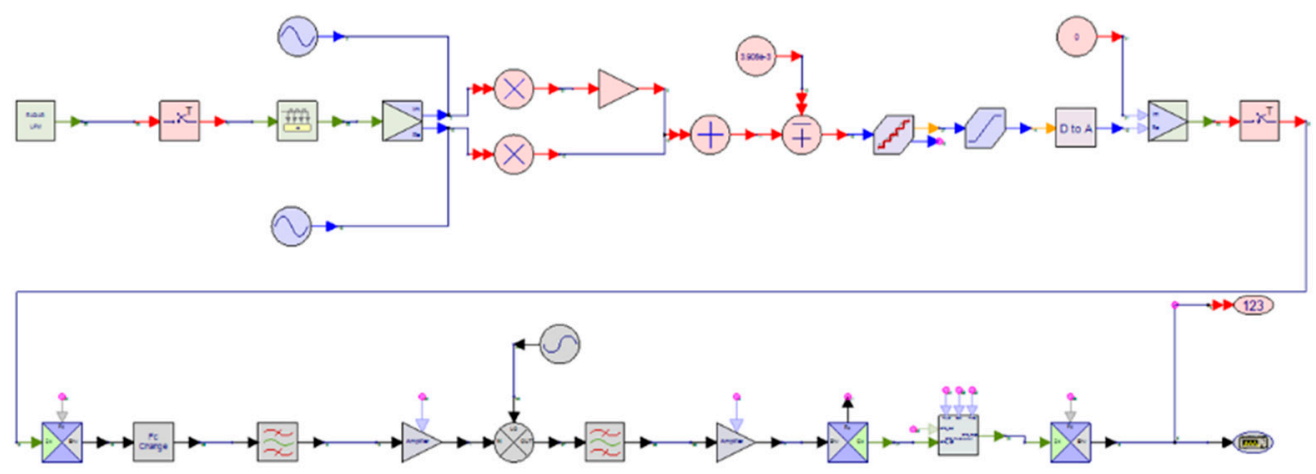

Figure 4. Radar system modeling.

\section{Experiments}

\subsection{Analysis and Verification of Fingerprint Characteristic}

The individual characteristics of the radar emitter are mainly reflected in the intrapulse unintentional modulation of the signal, which is mainly produced by the unwanted parasitic modulation generated by the frequency source oscillator, transmitting tube, mod- 
ulator, high voltage power supply and other devices or circuits of high-power radar transmitter [27-31]. Unintentional modulation is an inherent characteristic of high-power radar transmitter, which is difficult to eliminate completely. Moreover, its modulation form and modulation amount are different for different transmitters. Even each radar in the same batch of radars has different unintentional modulation distribution, because similar components still have slight differences in performance. Unintentional modulation always exists in radar signals and can reflect the individual differences of each radar. Because of the uniqueness and particularity of the unintentional modulation feature of each radar, the individual feature is also called "radar fingerprint". Many scholars have simulated the nonlinearity of radar emitter components, such as frequency source or amplifier. However, there is a lack of theoretical methods and mathematical models to systematically and comprehensively study the generation mechanism of "fingerprint" features from the overall nonlinearity of the radar emitter. Next, this model will simulate and verify the nonlinear characteristics of the important components in the typical radar emitter structure and analyze their comprehensive effects.

\subsubsection{Analysis and Verification of Nonlinear Characteristics of Signal Source}

There is always a carrier frequency in the radar emitter. Because any carrier frequency is not absolutely stable the actual radar emitter carrier frequency will not be completely equal to its nominal frequency value, and there is always more or less deviation. The phase noise and spurious output of frequency source of transmitting equipment are one of the main sources of spurious components of transmitting signal. The phase noise and spurious output of different radar emitters lead to different spurious components of different individual signals. There are many literatures on the analysis of phase noise and spurious output of crystal oscillator and PLL frequency source. Considering that direct digital synthesizer (DDS) has become the mainstream of modern radar emitter frequency synthesizer, this section mainly analyzes the phase noise, harmonic and spurious components of DDS output signal. Next, the spectrum structure, phase noise and spectrum spurious of the ideal DDS output signal are studied, and various factors causing the spurious of DDS output signal, such as phase truncation spurious and amplitude quantization spurious, are comprehensively analyzed.

Figure 5 shows the schematic diagram of DDS, the accumulator outputs linear increasing phase value, which is used as address to address sine function table. The output of ROM is converted into analog signal by DAC. In Figure 5, the word length of accumulator is $\mathrm{L}$, the value of frequency control word is $F_{C W}, \mathrm{~W}$ is the number of ROM address lines, $\mathrm{D}$ is the word length of ROM data line and D/A converter. The output frequency formula of DDS is:

$$
f_{\text {out }}=\frac{F_{C W}}{2^{N}} f_{c l k}
$$

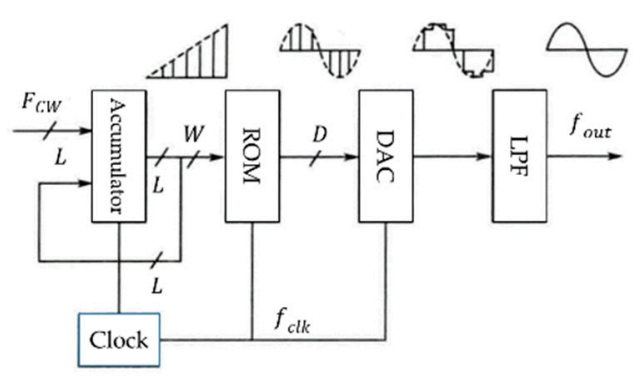

Figure 5. DDS schematic diagram.

There are two main spurious sources in actual DDS system: phase truncation error, and amplitude quantization error. These two spurious sources are discussed below.

I. Phase truncation error. 
According to Figure 5, it can be seen that the number of phase truncation bits $\mathrm{B}=\mathrm{L}-\mathrm{W}$. If only the spurious component caused by truncation is considered, only needs to analyze the output signal after phase to amplitude conversion. Assuming that there is no phase truncation, the discrete value of the output signal amplitude after addressing is:

$$
S(n)=\cos \left(2 \pi \frac{F_{C W}}{2^{L}} n\right)
$$

After B-bit truncation, the discrete value of output signal is Equation (2), where $F[x]$ is the downward integer of $x$.

$$
\mathrm{S}^{\prime}(n)=\cos \left(2 \pi \frac{2^{B}}{2^{L}} F\left[\frac{F_{C W}}{2^{B}} n\right]\right)
$$

Assuming that the phase rounding error is $\mathrm{e}_{p}(n)$, the above equation can be converted as follows, where $\langle X\rangle_{Y}$ means $X$ to $Y$ remainder operation.

$$
\begin{gathered}
\mathrm{S}^{\prime}(n)=\cos \left(\frac{2 \pi}{2^{L}}\left[F_{C W} n-\mathrm{e}_{p}(n)\right]\right) \\
\mathrm{e}_{p}(n)=F_{C W} n-2^{B} F\left[\frac{F_{C W}}{2^{B}} n\right]=\left\langle n F_{C W}\right\rangle_{2^{B}}
\end{gathered}
$$

The error sequence is analyzed as follows: since $\mathrm{e}_{p}(n)$ does not always return to zero after each overflow, it is assumed that the period of $\mathrm{e}_{p}(n)$ is $N_{k}$, then

$$
N_{k} F_{C W}=m 2^{B}
$$

Suppose $x$ is the greatest common divisor of $F_{C W}$ and $2^{B}$, then

$$
F_{C W}=x \cdot y, 2^{B}=x \cdot z\left(x, y, z \in N^{+}\right)
$$

It can be seen that the least common multiple of $F_{C W}$ and $2^{B}$ is $x \cdot y \cdot z=m 2^{B}=N_{k} F_{C W}$. Then $N_{k}=x y z / F_{C W}=2^{B} / x$, so the period of the error sequence $\mathrm{e}_{p}(n)$ is Equation (8), where formula $\operatorname{gcd}(x, y)$ means the greatest common divisor between $x$ and $y$.

$$
N_{k}=\frac{2^{B}}{\operatorname{gcd}\left(F_{C W}, 2^{B}\right)}
$$

According to the theory of discrete digital signal, the frequency spectrum of $\mathrm{e}_{p}(n)$ repeats with the clock frequency of $f_{c}$, then in the interval of $\left(0, f_{c} / 2\right]$, the frequency spectrum is composed of $2^{B-1} / \operatorname{gcd}\left(F_{C W}, 2^{B}\right)$ discrete spectral lines.

If the continuous form of the corresponding error after truncation is written as $\mathrm{e}_{p}(t)$, then $\varepsilon_{p}(n)$ can be considered as the result of sampling $\mathrm{e}_{p}(t)$ by clock cycle. It is easy to know that $\mathrm{e}_{p}(t)$ is a sawtooth wave with amplitude of $2^{B}$ and period of $2^{B} / F_{C W}$. In order to analyze the spectrum characteristics of $\mathrm{e}_{p}(t)$, we need to expand it by Fourier series, but because its value at the discontinuous point is 0 , it does not meet the Dirichlet condition, so it cannot be expanded directly. In order to solve this problem, Nicholas and Samueli propose a correction method: divide $\mathrm{e}_{p}(t)$ into two parts $e_{p}^{\prime}(t)$ and $\mathrm{p}(t)$ which satisfy the expansion condition, as shown in Figure 6, the period of $\mathrm{p}(t)$ is $T_{c} \cdot 2^{B} / F_{C W}$ and the pulse width is $T_{p}=\frac{\operatorname{gcd}\left(F_{C W}, 2^{B}\right)}{2^{B}}$, where:

$$
e_{p}^{\prime}(t)= \begin{cases}\mathrm{e}_{p}(t) & t \neq n T_{c} \cdot 2^{B} / F_{C W} \\ 2^{B-1} & t=n T_{c} \cdot 2^{B} / F_{C W}\end{cases}
$$




$$
\mathrm{p}(t)= \begin{cases}-2^{B-1} & t \in\left(n T_{c} \cdot 2^{B} / F_{C W}-\frac{T_{p}}{2}, n T_{c} \cdot 2^{B} / F_{C W}+\frac{T_{p}}{2}\right) \\ -2^{B-2} & t=n T_{c} \cdot 2^{B} / F_{C W} \pm \frac{T_{p}}{2} \\ 0 & \text { others }\end{cases}
$$

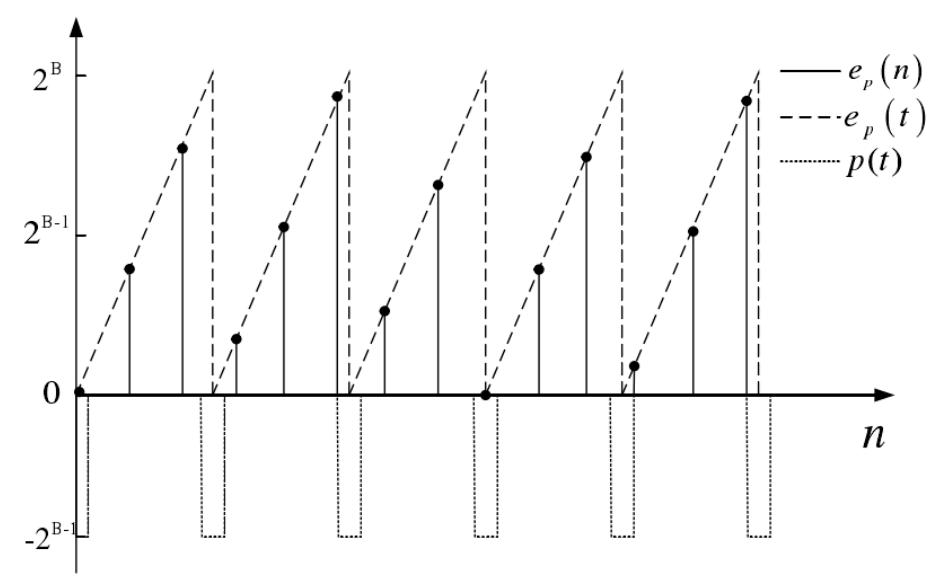

Figure 6. Phase truncation error sequence.

After the correction, the Fourier expansion of the above two equations can be obtained:

$$
\begin{aligned}
\mathrm{e}_{p}(t) & =e_{p}^{\prime}(t)+\mathrm{p}(t) \\
& =\sum_{k=1}^{+\infty} \frac{2^{B}}{\pi k} \sin \left(2 \pi k \frac{F_{C W}}{2^{B}} t\right)-\frac{2^{B}}{2 \Lambda} \sum_{k=1}^{+\infty} \frac{4 \Lambda}{2 \pi k} \sin \left(\frac{2 \pi k}{4 \Lambda}\right) \cos \left(2 \pi k \frac{F_{C W}}{2^{B}} t\right)
\end{aligned}
$$

Omit the DC component in the above formula, where $\Lambda=2^{B-1} / \operatorname{gcd}\left(F_{C W}, 2^{B}\right)$. The expansion is sampled as follows:

$$
\mathrm{e}_{p}(n)=\sum_{k=1}^{+\infty} \frac{2^{B}}{\pi k} \sin \left(2 \pi k \frac{F_{C W}}{2^{B}} n\right)-\frac{2^{B}}{2 \Lambda} \sum_{k=1}^{+\infty} \frac{4 \Lambda}{2 \pi k} \sin \left(\frac{2 \pi k}{4 \Lambda}\right) \cos \left(2 \pi k \frac{F_{C W}}{2^{B}} n\right)
$$

After detailed mathematical analysis of $\mathrm{e}_{p}(n)$, it can be expressed as the discrete weighted sum of $\Lambda$ spectral lines as follows:

$$
\mathrm{e}_{p}(n)=\sum_{k=1}^{\Lambda} \xi_{k} \exp \left(j 2 \pi k \frac{F_{C W}}{2^{B}} n\right) \exp (j \Psi(k, \Lambda))
$$

where $\xi_{k} \equiv \frac{2^{B}}{2 \Lambda} \csc \left(\frac{k \pi}{2 \Lambda}\right), \Psi(k, \Lambda) \equiv-\cot \left(\frac{k \pi}{2 \Lambda}\right)$ represent the amplitude and phase values of the $k$-th corresponding frequency component of the error vector respectively [32]. tion is:

According to $\frac{e_{p}(n)}{2^{L}} \leq \frac{2^{B}}{2^{L}} \ll 1$, the output signal sequence caused by phase trunca-

$$
S^{\prime}(n)=\cos \left(\frac{2 \pi}{2^{L}}\left[F_{C W} n-\mathrm{e}_{p}(n)\right]\right) \approx \cos \left(\frac{2 \pi}{2^{L}} F_{C W} n\right)+\frac{2 \pi}{2^{L}} \mathrm{e}_{p}(n) \sin \left(\frac{2 \pi}{2^{L}} F_{C W} n\right)
$$

It can be seen that the error sequence of output signal caused by phase truncation can be written as:

$$
\varepsilon_{p}(n)=S^{\prime}(n)-S(n) \approx \frac{2 \pi}{2^{L}} \mathrm{e}_{p}(n) \sin \left(\frac{2 \pi}{2^{L}} F_{C W^{n}} n\right)
$$

By substituting $\mathrm{e}_{p}(n)$ and the Fourier series expansion of $\sin \left(\frac{2 \pi}{2^{L}} F_{C W} n\right)$, we can get:

$$
\varepsilon_{p}(n) \approx \frac{2 \pi}{2^{L+1}} \sum_{k=1}^{\Lambda} \xi_{k} \exp \left(j \times\left(\Psi(k, \Lambda)+\frac{\pi}{2}\right)\right) \times\left(\exp \left(j \times \frac{2 \pi n F_{C W}}{2^{L}}\left(k 2^{L-B}+1\right)\right)-\exp \left(j \times \frac{2 \pi n F_{C W}}{2^{L}}\left(k 2^{L-B}-1\right)\right)\right)
$$


It can be seen from the above formula that the spurious component of the output signal is also composed of $\Lambda$ spectral lines. By mapping them to the actual frequency range of $\left(0, f_{c} / 2\right)$, the frequency value $f_{s k}$ and corresponding amplitude value $\xi_{s k}$ of each spectral line can be obtained as follows:

$$
\begin{gathered}
f_{s k}=f_{c} \times \min \left(\frac{\bmod \left(k F_{C W} 2^{L-B} \pm F_{C W}, 2^{L}\right)}{2^{L}}, 1-\frac{\bmod \left(k F_{C W} 2^{L-B} \pm F_{C W}, 2^{L}\right)}{2^{L}}\right) \\
\xi_{s k}=\frac{2 \pi}{2^{L+1}} \times \frac{2^{B-1}}{\Lambda} \times \csc \left(\frac{k \pi}{2 \Lambda}\right)=\frac{\pi \times 2^{B-L}}{2 \Lambda} \csc \left(\frac{k \pi}{2 \Lambda}\right)
\end{gathered}
$$

By further simplifying $\xi_{s k}$, we can get:

$$
\xi_{s k}=\frac{\pi 2^{B-L}}{2 \Lambda} \csc \left(\frac{k \pi}{2 \Lambda}\right)=2^{B-L} \frac{\pi \operatorname{gcd}\left(F_{C W}, 2^{B}\right) / 2^{B}}{\sin \left(\pi k g c d\left(F_{C W}, 2^{B}\right) / 2^{B}\right)}
$$

It is easy to see from the above formula that when $\mathrm{k}=1$, the corresponding frequency and amplitude values of the maximum stray frequency component are as follows:

$$
\begin{gathered}
f_{w s}=f_{c} \times \min \left(\frac{\bmod \left(F_{C W} 2^{L-B} \pm F_{C W}, 2^{L}\right)}{2^{L}}, 1-\frac{\bmod \left(F_{C W} 2^{L-B} \pm F_{C W}, 2^{L}\right)}{2^{L}}\right) \\
\xi_{w s}=2^{B-L} \frac{\pi \operatorname{gcd}\left(F_{C W}, 2^{B}\right) / 2^{B}}{\sin \left(\pi \operatorname{gcd}\left(F_{C W}, 2^{B}\right) / 2^{B}\right)}
\end{gathered}
$$

It can be further calculated that the SNR of single frequency output is:

$$
\mathrm{SNR}=20 \log \left(\frac{1}{\xi_{s k}}\right)=20 \log 2^{L-B}-20 \log \frac{\pi \operatorname{gcd}\left(F_{C W}, 2^{B}\right) / 2^{B}}{\sin \left(\pi k g c d\left(F_{C W}, 2^{B}\right) / 2^{B}\right)}
$$

Since $1<\frac{\pi \operatorname{gcd}\left(F_{C W}, 2^{B}\right) / 2^{B}}{\sin \left(\pi k g c d\left(F_{C W}, 2^{B}\right) / 2^{B}\right)}<\frac{\pi}{2}$, we can infer that:

$$
6.0206(L-B)-3.9224 \leq S N R \leq 6.0206(L-B)(d B)
$$

It can be seen that the spurious spectral line position of the output signal caused by the phase truncation of the direct digital waveform synthesis method is determined by the input frequency control word $F_{C W}$ and the word length of the phase accumulator $L$, and the spurious level is determined by the value of $L-B$.

Based on the phase truncation of DDS system, the simulation of single frequency signal can verify that the spurious characteristics meet the above conclusions. The simulation parameters are set as shown in Table 1, and the simulation results are shown in Figure 7.

Table 1. Parameter of the direct digital synthesizer.

\begin{tabular}{cc}
\hline Parameter & Value \\
\hline Phase accumulator bits & $32 \mathrm{bits}$ \\
Phase truncation bits & $16 \mathrm{bits}$ \\
Output frequency & $7.7 \mathrm{MHz}$ \\
System sampling rate & $20 \mathrm{MHz}$ \\
\hline
\end{tabular}

According to the above analysis of the phase truncation spurious component, substituting the parameters into Equations (20) and (21), $f_{w s}=0.5 \mathrm{MHz}$ and $\xi_{w s}=96.31 \mathrm{~dB}$ can be calculated. It can be seen that the simulation results are basically consistent with the theoretical derivation. 


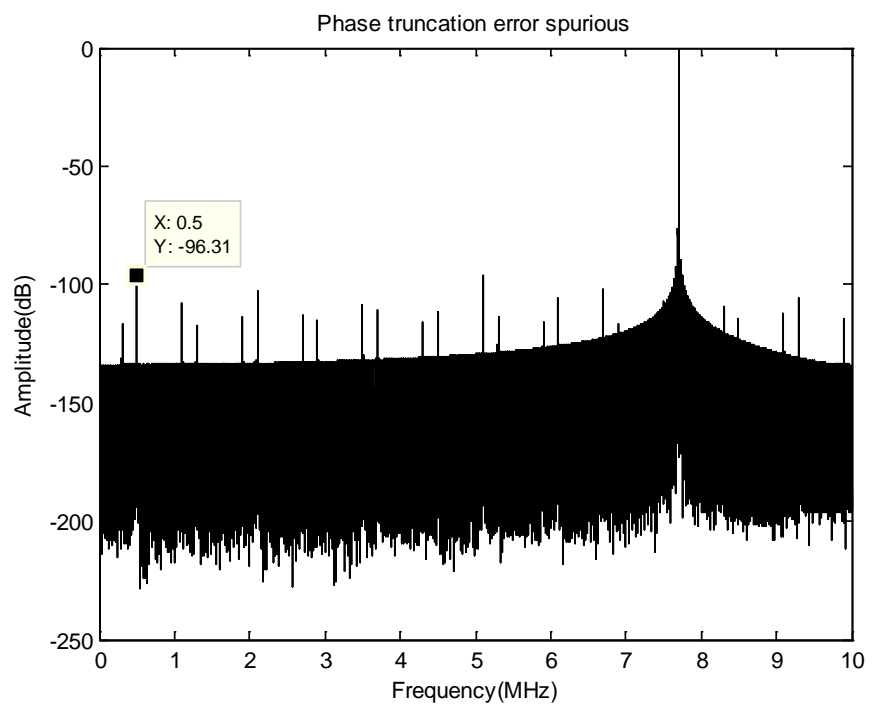

Figure 7. Phase truncation error spurious.

According to the digital characteristics of the direct digital frequency synthesis method, the frequency of the LFM signal generated by the direct digital frequency synthesis method is increasing step by step, so all the frequency components of the signal in the whole frequency band can be regarded as the superposition of the spurious components of each single frequency component. Since the frequency band range of DDS output signal is within $f_{c} / 2$ at most, the maximum spurious frequency corresponding to each frequency component in this range can be calculated one by one. As the output frequency increases, the maximum spurious frequency changes, as shown in Figure 8 . The parameters are set as $\mathrm{L}=24, \mathrm{~B}=18$.

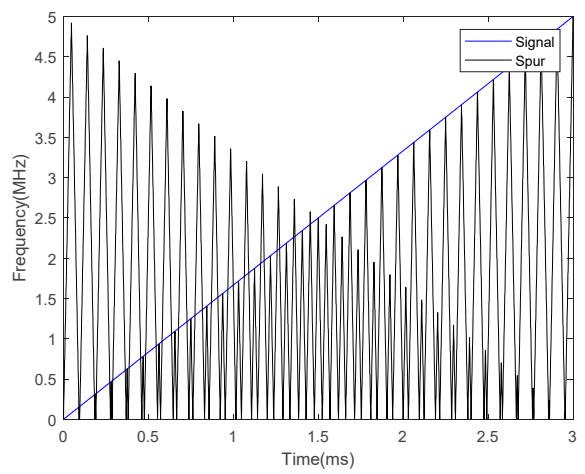

Figure 8. Frequency relation diagram of signal and spurious.

It can be seen from Figure 8 that as the frequency of the output signal increases linearly with time, the corresponding maximum stray frequency also changes linearly with time, but the change rate is far greater than the frequency modulation slope of the signal, showing an irregular sawtooth wave. Calculate the frequency modulation slope $k_{\text {sig }}=1.67 e^{-4}$ and $k_{\text {spur }}=1.08 e^{-2}$ of signal and spurious respectively, define $r_{k}=\frac{k_{\text {spur }}}{k_{\text {sig }}}$ to represent the change rate relationship between signal and spurious, it can be seen that when L-B $=6$, the change slope of spurious is 65 times of that of signal, which is much larger than that of signal. Through simulation, it can be found that $r_{k}$ changes with the change of L-B, and the overall change trend is shown in Figure 9. 


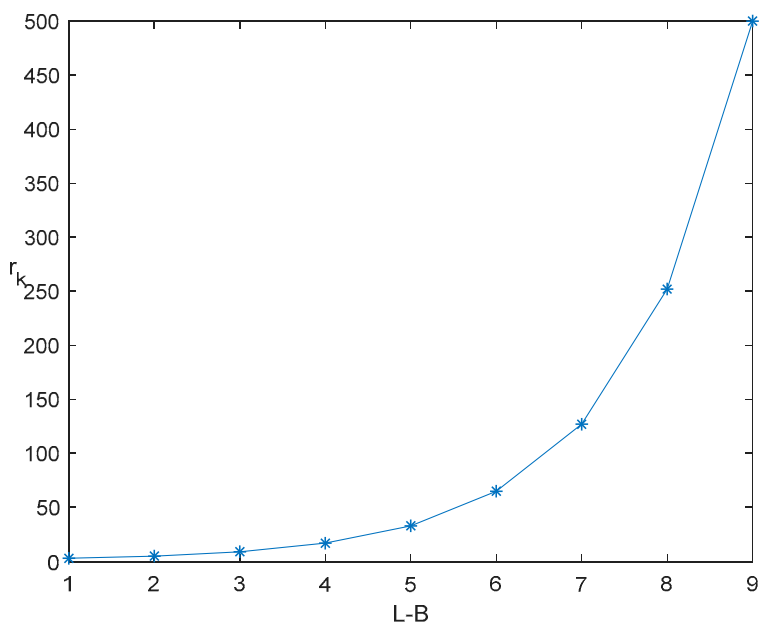

Figure 9. Relationship between $r_{k}$ and L-B.

It is not difficult to see that with the increase of $\mathrm{L}-\mathrm{B}, r_{k}$ shows an exponential growth trend. Therefore, when the L-B value is large, the spurious bandwidth will be far greater than the signal bandwidth, and the larger the L-B value is, the more uniform the spurious distribution is. After filtering, the spurious distribution in the band is approximately uniform relative to the signal.

In a word, it can be considered that the spurious of DDS signal source due to phase truncation mainly reduces the signal-to-noise ratio of LFM signal source. Based on DDS phase truncation, the LFM signal is simulated, and the simulation parameters are set as shown in Table 2.

From Equation (23), the signal-to-noise ratio of the output signal is $32 \mathrm{~dB} \leq S N R \leq 36 \mathrm{~dB}$. The spectrum of the output signal is shown in Figure 10. From the simulation results, it can be seen that the spurious component is reflected in the form of noise, and the simulation results are basically consistent with the theory.

Table 2. Parameter of direct digital synthesizer.

\begin{tabular}{cc}
\hline Parameter & Value \\
\hline System sampling rate & $50 \mathrm{MHz}$ \\
Phase accumulator bits & $32 \mathrm{bits}$ \\
Phase truncation bits & $26 \mathrm{bits}$ \\
Pulse repetition period & $100 \mu \mathrm{s}$ \\
Pulse width & $20 \mu \mathrm{s}$ \\
Starting frequency & $2 \mathrm{MHz}$ \\
Termination frequency & $5 \mathrm{MHz}$ \\
\hline
\end{tabular}

II. Amplitude quantization error.

Since amplitude quantization occurs after phase truncation, the distribution characteristics of spurious signals caused by amplitude quantization are discussed in two cases: with and without phase truncation. Here we define $F_{C W} / 2^{N}=v_{k} / u_{k}=f_{0} / f_{c}\left(v_{k}\right.$ and $u_{k}$ are prime to each other), the two cases are discussed as follows:

When $F_{C W}=k \cdot 2^{B}$ and there is no phase truncation, the spurious caused by quantization error can be expressed as follows:

$$
e_{q}(n)=\cos \left(2 \pi \frac{n v_{k}}{u_{k}}\right)-\frac{1}{2^{D-1}} i n t\left[2^{D-1} \cos \left(2 \pi \frac{n v_{k}}{u_{k}}\right)\right]
$$




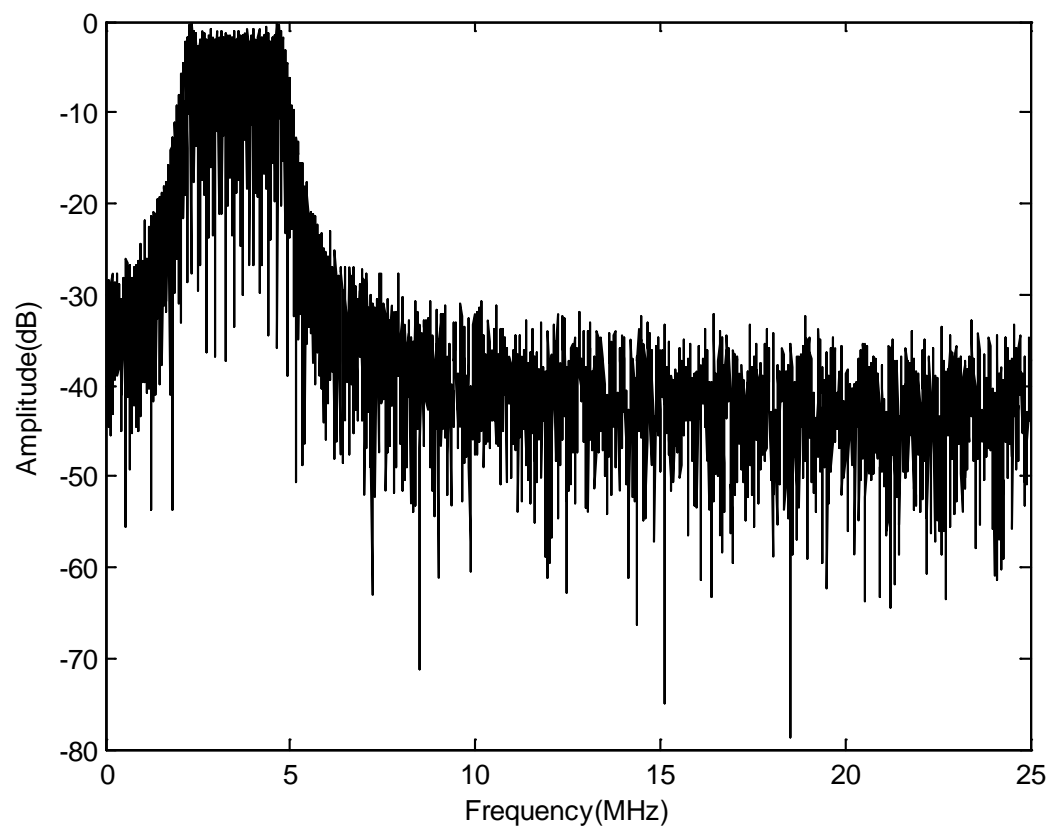

Figure 10. Spectrum of truncated LFM signal.

It can be seen that $e_{q}(n)$ is a periodic sequence with a period of $\frac{u_{k}}{\operatorname{gcd}\left(u_{k}, v_{k}\right)}=u_{k}$, so the output spurious signal has at most $u_{k}$ frequency components in the $\left(0, f_{c}\right)$ interval. Therefore, the kth frequency value of the output spurious signal should be:

$$
f_{k}=\frac{k f_{c}}{u_{k}}=\frac{k g c d\left(2^{N}, F_{C W}\right)}{F_{C W}} f_{0}=\frac{k g c d\left(f_{0}, f_{c}\right)}{F_{C W}} f_{0}
$$

It can be proved that when $\mathrm{k}$ is even, the amplitude at frequency $f_{k}$ is 0 , so the spurious signal only contains the odd multiple frequency harmonic of $\frac{f_{c}}{u_{k}}$. The simulation parameter settings are shown in Table 3.

Table 3. Parameter of direct digital synthesizer.

\begin{tabular}{cc}
\hline Parameter & Value \\
\hline System sampling rate & $20 \mathrm{MHz}$ \\
Phase accumulator bits & $32 \mathrm{bits}$ \\
Phase truncation bits & $26 \mathrm{bits}$ \\
Amplitude quantization bits & 5 \\
Input frequency control word & $469,762,048$ \\
\hline
\end{tabular}

Here $F_{C W}=7 \times 2^{26}=469,762,048, u_{k}=2^{6}=64$. It can be calculated that the frequency value of the output signal $f_{0}=\frac{F_{C W}}{2^{N}} f_{c}=2.1875 \mathrm{MHz}$, and the corresponding quantization spurious is $(2 k+1) \frac{f_{c}}{u_{k}}=(2 k+1) \times 0.3125 \mathrm{MHz}$. The simulation results are shown in Figure 11. It can be seen that the position and distribution of stray components are consistent with the theory.

When $F_{C W} \neq k \cdot 2^{B}$, the phase truncation error occurs in the front end. According to Equation (3), the spurious component generated by quantization error can be expressed as:

$$
e_{p q}(n)=\cos \left(2 \pi \frac{2^{B}}{2^{L}} F\left[\frac{F_{C W}}{2^{B}} n\right]\right)-\frac{1}{2^{D-1}} i n t\left[2^{D-1} \cos \left(2 \pi \frac{2^{B}}{2^{L}} F\left[\frac{F_{C W}}{2^{B}} n\right]\right)\right]
$$




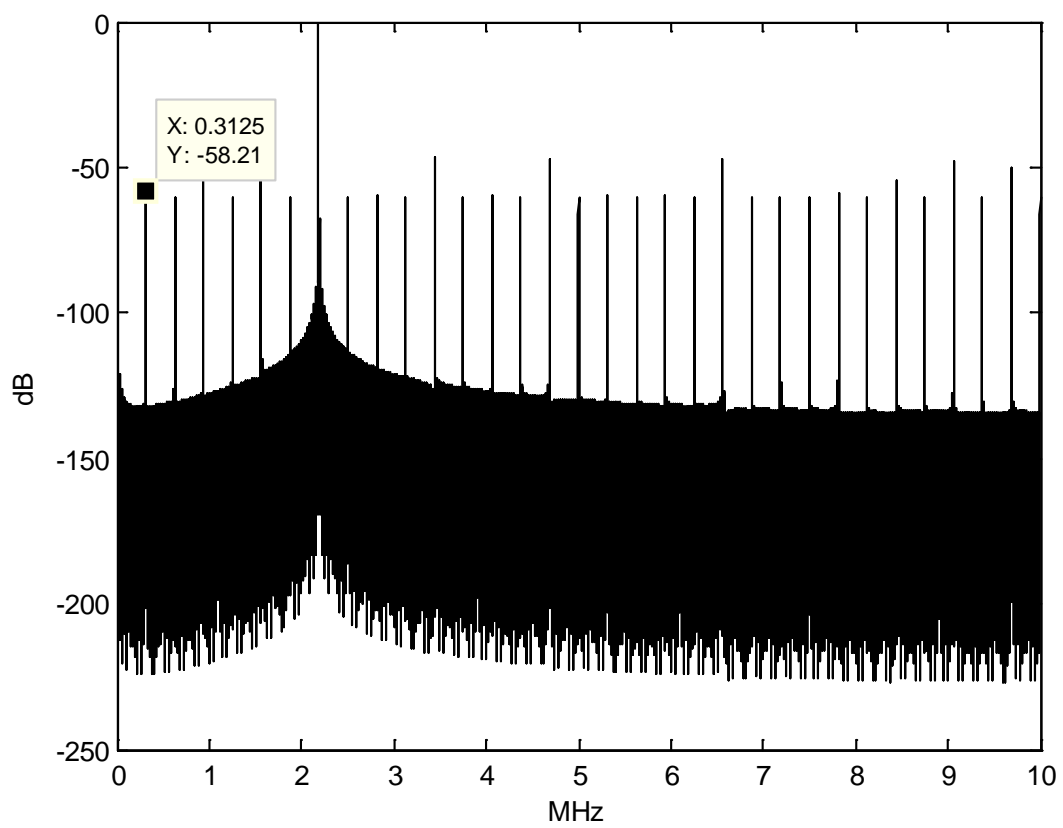

Figure 11. Amplitude quantization error spurious without phase truncation.

According to the previous formula:

$$
S^{\prime}(n)=\cos \left(\frac{2 \pi}{2^{L}}\left[F_{C W} n-\mathrm{e}_{p}(n)\right]\right) \approx \cos \left(\frac{2 \pi}{2^{L}} F_{C W^{n}}\right)+\frac{2 \pi}{2^{L}} \mathrm{e}_{p}(n) \sin \left(\frac{2 \pi}{2^{L}} F_{C W} n\right)
$$

Then

$$
e_{p q}(n)=S^{\prime}(n)-\frac{1}{2^{D-1}} i n t\left[2^{D-1} S^{\prime}(n)\right]
$$

It is not difficult to see that the longest period of $e_{p q}(n)$ can be consistent with that of $S^{\prime}(n)$, which is $\frac{2^{N}}{\operatorname{gcd}\left(2^{N}, F_{C W}\right)}$. At this time, the period of amplitude quantization spurious signal is longer, and the corresponding distribution in frequency domain is more dispersed. When $F_{C W}$ is odd, the period of $e_{p q}(n)$ can reach $2^{N}$. If $2^{N}$ points DFT is performed on the signal, the spurious noise will be distributed on each spectral line, which can be considered as approximately uniformly distributed noise.

Considering the influence of amplitude quantization in the above two cases, it can be seen that when the LFM signal is output, because the frequency component of the output signal will occupy a certain bandwidth, the amplitude quantization spurious distribution corresponding to each different frequency value is also different.

Next, we analyze the noise power value caused by amplitude quantization, the amplitude quantization error is regarded as a random variable obeying uniform distribution in the range of $\left[-\frac{\Delta}{2}, \frac{\Delta}{2}\right]$, where $\Delta=2^{-D+1}$ and $D$ is the quantization number. $x$ is the sine function before quantization, $x_{q}$ is the sine function after quantization, and $e_{q}(n)=x-x_{q}$ is the amplitude quantization error function. After digital to analog conversion, the amplitude quantization error function becomes a step waveform. Considering its periodicity, the time domain expression of its signal in one cycle is obtained as follows:

$$
e_{q}(t)=\sum_{k=0}^{u_{k}-1} e_{q}(k)\left[u\left(t-k T_{c}\right)-u\left(t-(k+1) T_{c}\right)\right]
$$


where $u(t)$ is the unit step function. The average power of $e_{q}(t)$ can be calculated as:

$$
\bar{P}=\frac{1}{T_{k}} \int_{0}^{T_{k}} e_{q}^{2}(t) d t=\frac{1}{T_{k}} \sum_{k=0}^{u_{k}-1} \int_{k T_{c}}^{(k+1) T_{c}} e_{q}^{2}(k) d t=\frac{1}{u_{k}} \sum_{k=0}^{u_{k}-1} e_{q}^{2}(k)
$$

Here, $e_{q}(k)$ is regarded as a uniform distribution in the range of $\left[-\frac{\Delta}{2}, \frac{\Delta}{2}\right]$, so it is more convenient to calculate its average power in a statistical sense as follows:

$$
\mathrm{E}[\bar{P}]=\frac{1}{u_{k}} \sum_{k=0}^{u_{k}-1} E\left(e_{q}^{2}(k)\right)=\frac{\Delta^{2}}{12}
$$

Assuming that the amplitude of the output signal is 1, the total SNR of the output signal can be expressed as:

$$
\mathrm{SNR}=10 \log \left(\frac{6}{\Delta^{2}}\right)=10 \log 6+20(D-1) \log 2=6.02 \mathrm{D}+1.76(d B)
$$

Therefore, the total SNR of output signal amplitude quantization noise is only related to quantization level bits. Considering the phase truncation effect and amplitude quantization effect, the output LFM signal is simulated, and the parameter settings are shown in Table 4.

Table 4. Parameter of direct digital synthesizer.

\begin{tabular}{cc}
\hline Parameter & Value \\
\hline System sampling rate & $50 \mathrm{MHz}$ \\
Phase accumulator bits & $32 \mathrm{bits}$ \\
Phase truncation bits & $26 \mathrm{bits}$ \\
Amplitude quantization bits & $8 \mathrm{bits}$ \\
Pulse repetition period & $100 \mu \mathrm{s}$ \\
Pulse width & $20 \mu \mathrm{s}$ \\
Starting frequency & $2 \mathrm{MHz}$ \\
Termination frequency & $5 \mathrm{MHz}$ \\
\hline
\end{tabular}

According to the previous theoretical analysis, the amplitude quantization of the phase truncated signal is carried out. When the quantization bit is 8 , the spectrum and amplitude quantization noise of the output LFM signal are shown in Figure 12. The SNR caused by the amplitude quantization is about $50 \mathrm{~dB}$, which is consistent with the theoretical calculation results.

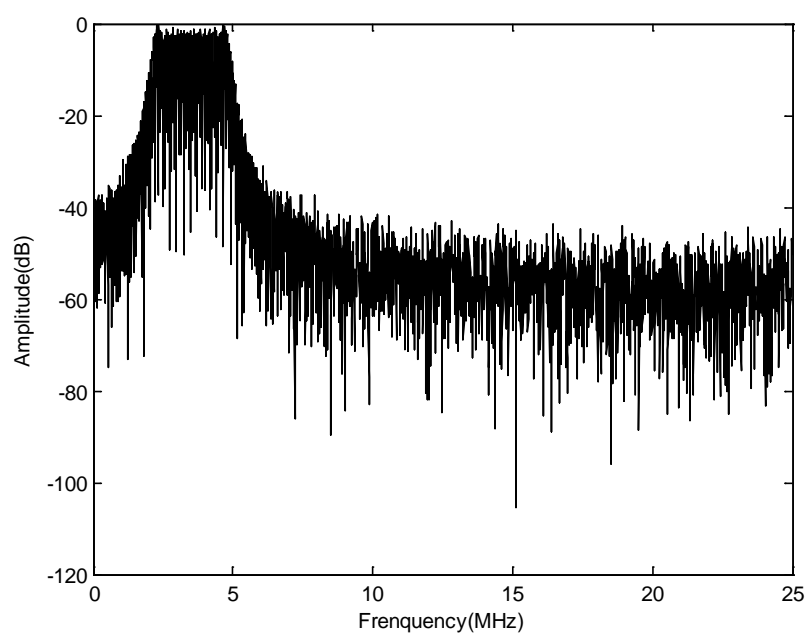

Figure 12. LFM signal spectrum after amplitude quantization. 


\subsubsection{Analysis and Verification of Nonlinear Characteristics of Mixer and Amplifier}

The mixer and amplifier are the core modules in the transmission channel. The mixer is responsible for moving the IF signal spectrum to the RF region, and the amplifier is responsible for amplifying the input RF signal. However, the nonlinear characteristic models of the two are consistent, as shown in Figure 13. The nonlinear characteristic parameters mainly include $1 \mathrm{~dB}$ compression point, third-order truncation point and second-order truncation point [33-35]. The nonlinear effects are mainly high-order harmonics and intermodulation distortion. Next, we analyze them respectively.

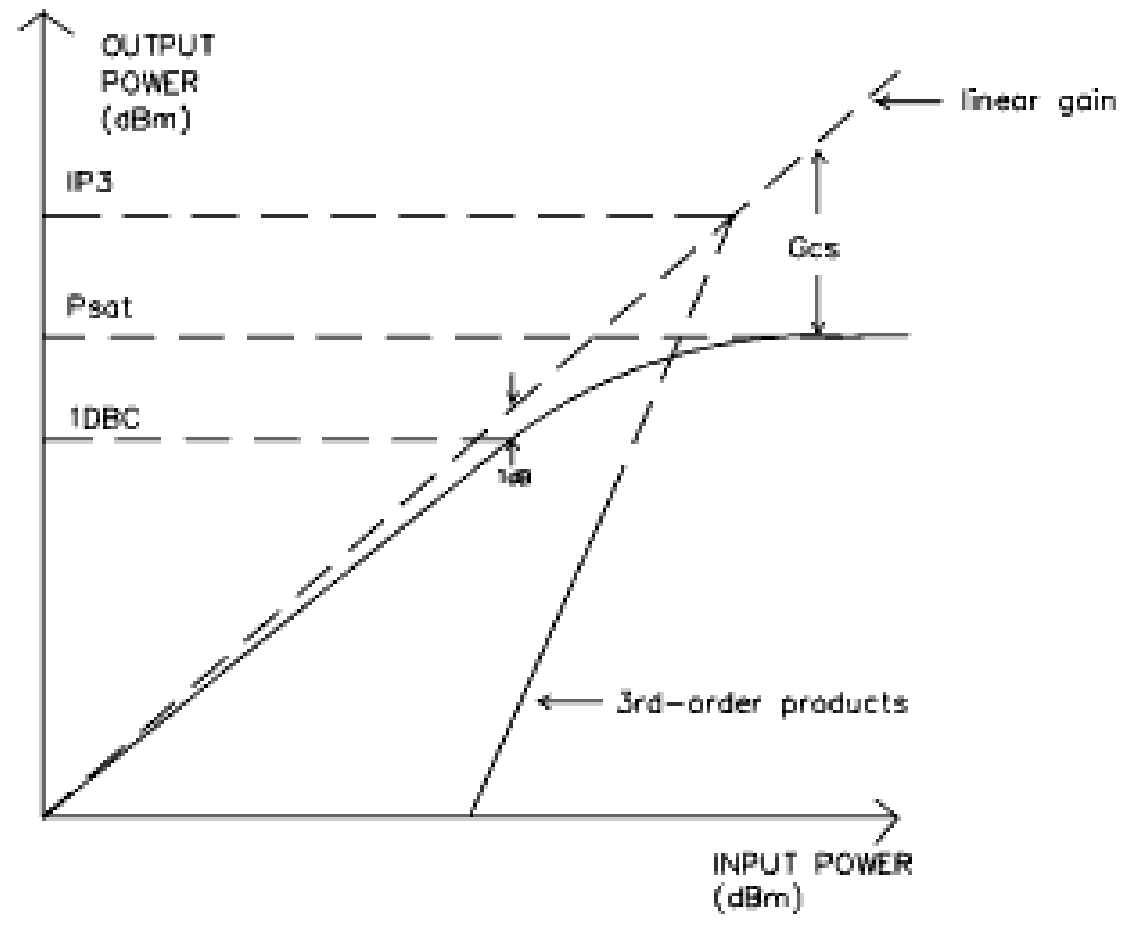

Figure 13. Schematic diagram of nonlinear characteristic index.

Assuming that the fourth-order and higher-order harmonics generated by nonlinearity can be ignored, the input nonlinearity can be expressed by a third-order power series. The relationship between input and output is as follows:

$$
v_{o}=a_{1} v_{i}+a_{2} v_{i}^{2}+a_{3} v_{i}^{3}
$$

Among them, $v_{i}$ and $v_{0}$ respectively represent the signal amplitude at the input and the output. When the input signal is a single frequency signal:

$$
v_{i}(t)=v \cos (\omega t)
$$

The output signal can be expressed as:

$$
\begin{aligned}
& v_{0}(t)=a_{1}[v \cos (\omega t)]+a_{2}[v \cos (\omega t)]^{2}+a_{3}[v \cos (\omega t)]^{3} \\
& =a_{1} v \cos (\omega t)+a_{2} v^{2} \cdot \frac{1}{2}[1+\cos (2 \omega t)]+a_{3} v^{3} \cdot \frac{1}{4}[3 \cos (\omega t)+\cos (3 \omega t)] \\
& =\frac{1}{2} a_{2} v^{2}+\left(a_{1} v+\frac{3}{4} a_{3} v^{3}\right) \cdot \cos (\omega t)+\frac{1}{2} a_{2} v^{2} \cos (2 \omega t)+\frac{1}{4} a_{3} v^{3} \cos (3 \omega t)
\end{aligned}
$$

It can be seen from the above formula that when the input is a single frequency signal, under the influence of nonlinear effect, compared with the power spectrum of the input signal, it is found that the power at different frequency points on the fundamental and adjacent channels is greater than that of the input signal, which is called spectrum growth [36]. The output signal will produce the second and third harmonic of the funda- 
mental frequency signal in addition to the fundamental frequency signal. The relationship between the coefficients of each component can be shown in Table 5 .

Table 5. Nonlinear coefficients of harmonic components.

\begin{tabular}{cccc}
\hline & $a_{1} v$ & $a_{2} v^{2}$ & $a_{3} v^{3}$ \\
\hline $\mathrm{DC}$ & - & $1 / 2$ & - \\
$\omega$ & 1 & - & $3 / 4$ \\
$2 \omega$ & - & $1 / 2$ & - \\
$3 \omega$ & - & - & $3 / 4$ \\
\hline
\end{tabular}

When the input is a dual frequency signal, the output signal will be generated according to the following rules:

$$
\begin{aligned}
& v_{o}(t)=a_{1}\left[v_{1} \cos \left(\omega_{1} t\right)+v_{2} \cos \left(\omega_{2} t\right)\right]+a_{2}\left[v_{1} \cos \left(\omega_{1} t\right)+v_{2} \cos \left(\omega_{2} t\right)\right]^{2}+a_{3}\left[v_{1} \cos \left(\omega_{1} t\right)+v_{2} \cos \left(\omega_{2} t\right)\right]^{3} \\
& =\frac{1}{2} a_{2}\left(v_{1}^{2}+v_{2}^{2}\right)+\left[a_{1} v_{1}+a_{3}\left(\frac{3}{4} v_{1}^{3}+\frac{3}{2} v_{1} v_{2}^{2}\right)\right] \cos \left(\omega_{1} t\right)+\left[a_{1} v_{2}+a_{3}\left(\frac{3}{4} v_{2}^{3}+\frac{3}{2} v_{1}^{2} v_{2}\right)\right] \cos \left(\omega_{2} t\right) \\
& +\frac{1}{2} a_{2} v_{1}^{2} \cos \left(2 \omega_{1} t\right)+\frac{1}{2} a_{2} v_{2}^{2} \cos \left(2 \omega_{2} t\right)+a_{2} v_{1} v_{2} \cos \left[\left(\omega_{1}+\omega_{2}\right) t\right]+a_{2} v_{1} v_{2} \cos \left[\left(\omega_{1}-\omega_{2}\right) t\right] \\
& +\frac{3}{4} a_{3} v_{1}^{2} v_{2} \cos \left[\left(2 \omega_{1}-\omega_{2}\right) t\right]+\frac{3}{4} a_{3} v_{1} v_{2}^{2} \cos \left[\left(2 \omega_{2}-\omega_{1}\right) t\right]+\frac{3}{4} a_{3} v_{1}^{2} v_{2} \cos \left[\left(2 \omega_{1}+\omega_{2}\right) t\right]+\frac{3}{4} a_{3} v_{1} v_{2}^{2} \cos \left[\left(2 \omega_{2}+\omega_{1}\right) t\right] \\
& +\frac{1}{4} a_{3} v_{1}^{3} \cos \left(3 \omega_{1} t\right)+\frac{1}{4} a_{3} v_{2}^{3} \cos \left(3 \omega_{2} t\right)
\end{aligned}
$$

It can be seen that the output signal not only has the required main signal components, but also produces DC components, second-order and third-order harmonics and the corresponding intermodulation components of second-order and third-order. The coefficients of each component are summarized in Table 6.

Table 6. Nonlinear coefficients of harmonic and intermodulation components.

\begin{tabular}{cccccccccc}
\hline & $a_{1} v_{1}$ & $a_{1} v_{2}$ & $a_{2} v_{1} v_{2}$ & $a_{2} v_{1}^{2}$ & $a_{2} v_{2}^{2}$ & $a_{3} v_{1} v_{2}^{2}$ & $a_{3} v_{1}^{2} v_{2}$ & $a_{3} v_{1}^{3}$ & $a_{3} v_{2}^{3}$ \\
\hline $\mathrm{DC}$ & - & - & - & $1 / 2$ & $1 / 2$ & - & - & - & - \\
$\omega_{1}$ & 1 & - & - & - & - & $3 / 2$ & - & $3 / 4$ & - \\
$\omega_{2}$ & - & 1 & - & - & - & - & $3 / 2$ & - & $3 / 4$ \\
$2 \omega_{1}$ & - & - & - & $1 / 2$ & - & - & - & - & - \\
$2 \omega_{2}$ & - & - & - & - & $1 / 2$ & - & - & - & - \\
$\omega_{1} \pm \omega_{2}$ & - & - & 1 & - & - & - & - & - & - \\
$2 \omega_{1} \pm \omega_{2}$ & - & - & - & - & - & - & $3 / 4$ & - & - \\
$2 \omega_{2} \pm \omega_{1}$ & - & - & - & - & - & $3 / 4$ & - & - & - \\
$3 \omega_{1}$ & - & - & - & - & - & - & - & - \\
$3 \omega_{2}$ & - & - & - & - & - & - & - & - & $1 / 4$ \\
\hline
\end{tabular}

Then, the nonlinear characteristics of $1 \mathrm{~dB}$ compression point, third-order truncation point and second-order truncation point are analyzed and verified.

I. $1 \mathrm{~dB}$ compression point $P_{\text {in }-1 \mathrm{~dB}}$

Due to the nonlinearity of the input channel, when the input signal power reaches $P_{\text {in }-1 d B}$, the power gain of the output signal relative to the input signal will decrease $1 \mathrm{~dB}$. The calculation of $1 \mathrm{~dB}$ compression point is deduced as follows: under the assumption of linear condition, the signal before and after entering the analog device has the following relationship:

$$
v_{o}=a_{1} v_{i}=a_{1} v_{i n-1 d B} \cos (\omega t)
$$

That is, the amplitude of the fundamental frequency component of the output signal is $a_{1} v_{i n-1 d B}$. When there is nonlinearity, according to the above analysis, the amplitude of the output fundamental frequency component should be $a_{1} v_{i n-1 d B}+\frac{3}{4} a_{3} v_{i n-1 d B}^{3}$. According to the definition of $P_{i n-1 d B}$, it can be calculated by $20 \cdot \log _{10}\left(10^{-0.05}\right)=-1 d B$. Let 
$a_{1} v_{i n-1 d B}+\frac{3}{4} a_{3} v_{i n-1 d B}^{3}=a_{1} v_{i n-1 d B} \cdot 10^{-0.05}$, the power of the input signal can be expressed as:

$$
P_{i n-1 d B}=v_{\text {in }-1 d B}^{2}=\frac{4 a_{1}\left(1-10^{-0.05}\right)}{3 a_{3}}
$$

This shows that when $1 \mathrm{~dB}$ gain compression occurs in the input, the amplitude of the received sinusoidal waveform will be $v_{i n-1 d B}$, and the corresponding power value will be $P_{i n-1 d B}$. It can be seen that the $1 \mathrm{~dB}$ compression point of the system is due to the increasing proportion of the harmonic component at the output end of the system and the corresponding reduction of the energy of the fundamental frequency component, resulting in the gain compression phenomenon.

II. Third order truncation point $\mathrm{IIP}_{3}$

When the dual frequency signal is input, the power of the output third-order intermodulation signal caused by nonlinearity is equal to the power of the output fundamental frequency signal, and the corresponding input signal power level is $\mathrm{IIP}_{3}$. The following is a quantitative derivation of the expression of the third-order truncation point: Assuming that the corresponding waveform amplitude is $v_{p 3}$ when the input reaches the third-order truncation point, according to the definition of $\mathrm{IIP}_{3}$ and the corresponding coefficient of the third-order intermodulation component obtained from the above analysis, we can get the following results:

$$
a_{1} v_{p 3}=\frac{3}{4} a_{3} v_{p 3}^{3}
$$

The power of the input signal can be expressed as:

$$
\mathrm{IIP}_{3}=v_{p 3}^{2}=\frac{4 a_{1}}{3 a_{3}}
$$

It can be seen that the value of the third-order truncation point mainly depends on the gain coefficient of the output fundamental frequency component and the gain coefficient of the third-order harmonic, the quantitative relationship between this index and $1 \mathrm{~dB}$ compression point can be calculated as follows:

$$
v_{p 3}^{2}=\frac{4}{3} \cdot \frac{3 v_{i n-1 d B}^{2}}{4\left(1-10^{-0.05}\right)}
$$

Taking logarithm of the above formula, we can get the following results:

$$
\mathrm{IIP}_{3}=P_{\text {in }-1 d B}+9.6(d B)
$$

Furthermore, the relationship between the input signal power level and the thirdorder intermodulation component, and the third-order harmonic component level in the linear range can be calculated. When the power level of the input signal is $P_{i}$, the conversion gain is $\mathrm{G}$, and the amplitude of the input signal is $v_{i}$, the amplitude of the corresponding fundamental frequency can be approximately considered as $a_{1} v_{i}$. At this time, the amplitude of the third-order intermodulation component generated in the output signal is:

$$
\frac{3}{4} a_{3} v_{i}^{3}=\frac{3}{4} v_{i}^{3} \cdot \frac{4 a_{1}}{3 v_{p 3}^{2}}=\frac{a_{1} v_{i}^{3}}{v_{p 3}^{2}}
$$

When converted to $\mathrm{dBm}$, the following results can be obtained:

$$
\mathrm{IM} 3=3 P_{i}+G-2 I I P_{3}
$$

The suppression degree of the third-order intermodulation component relative to the fundamental frequency component at the corresponding output is as follows:

$$
\Delta_{3}=2 P_{i}-2 I I P_{3}
$$


According to the analysis of the third-order harmonic component, the corresponding output third-order harmonic level is $\frac{1}{4} a_{3} v_{i}^{3}$, so the third-order harmonic level is:

$$
\mathrm{HM} 3=\mathrm{IM} 3-20 \log _{10} 3=\mathrm{IM} 3-9.54(d B)
$$

The output third-order harmonic suppression is:

$$
\Delta_{h 3}=\Delta_{3}+9.54(d B)
$$

III. Second order truncation point $\mathrm{IIP}_{2}$

When the dual frequency signal is input, the power of the output second-order intermodulation signal caused by nonlinearity is equal to the power of the output fundamental frequency signal, and the corresponding input signal power level is $\mathrm{IIP}_{2}$. The following is a quantitative derivation of the expression of the second-order truncation point: Assuming that the corresponding waveform amplitude is $v_{p 2}$ when the input reaches the second-order truncation point, according to the definition of $\mathrm{IIP}_{2}$ and the corresponding coefficient of the second-order intermodulation component obtained from the above analysis, we can get the following results:

$$
a_{2} v_{p 2}^{2}=\frac{3}{4} a_{1} v_{p 2}
$$

The power of the input signal can be expressed as:

$$
\mathrm{IIP}_{2}=v_{p 2}^{2}=\frac{a_{1}^{2}}{a_{2}^{2}}
$$

Furthermore, the relationship between the input signal power level and the secondorder intermodulation component, and the second-order harmonic component level in the linear range can be calculated. When the power level of the input signal is $P_{i}$, the conversion gain is $\mathrm{G}$, and the amplitude of the input signal is $v_{i}$, the amplitude of the corresponding fundamental frequency can be approximately considered as $a_{1} v_{i}$. At this time, the amplitude of the second-order intermodulation component generated in the output signal is:

$$
a_{2} v_{i}^{2}=\frac{a_{1} v_{i}^{2}}{v_{p 2}}
$$

When converted to $\mathrm{dBm}$, the following results can be obtained:

$$
\mathrm{IM} 2=2 P_{i}+G-I I P_{2}
$$

The suppression degree of the second-order intermodulation component relative to the fundamental frequency component at the corresponding output is as follows:

$$
\Delta_{2}=P_{i}-I I P_{2}
$$

According to the analysis of the second-order harmonic component, the corresponding output second-order harmonic level is $\frac{1}{2} a_{2} v_{i}^{2}$, so the second-order harmonic level is:

$$
\mathrm{HM} 2=\mathrm{IM} 2-20 \log _{10} 2=\mathrm{IM} 2-6(d B)
$$

The output second-order harmonic suppression is:

$$
\Delta_{h 2}=\Delta_{2}+6(d B)
$$

According to the analysis of DDS in the previous paper, the IF output signal after ideal orthogonal modulation only contains useful signal components and noises of various components, as shown in Figure 14. After mixing, amplifying and other processing, the RF output signal component should include the LO, useful signal and all order harmonics of 
the useful signal-mainly, the second-order and third-order harmonics. If the IF signal is expressed as:

$$
S_{I F}(t)=\exp \left(2 \pi f_{I F} t+k \pi t^{2}\right)
$$

Then according to the nonlinear characteristic model of mixer and amplifier, the RF signal can be expressed as:

$$
\begin{gathered}
S_{R F}(t)=a_{1} \exp \left(2 \pi f_{L O} t+2 \pi f_{I F} t+k \pi t^{2}\right)+\frac{1}{2} a_{2} \exp \left(2 \pi f_{L O} t+2\left(2 \pi f_{I F} t+k \pi t^{2}\right)\right) \\
+\frac{1}{4} a_{3} \exp \left(2 \pi f_{L O} t+3\left(2 \pi f_{I F} t+k \pi t^{2}\right)\right)
\end{gathered}
$$

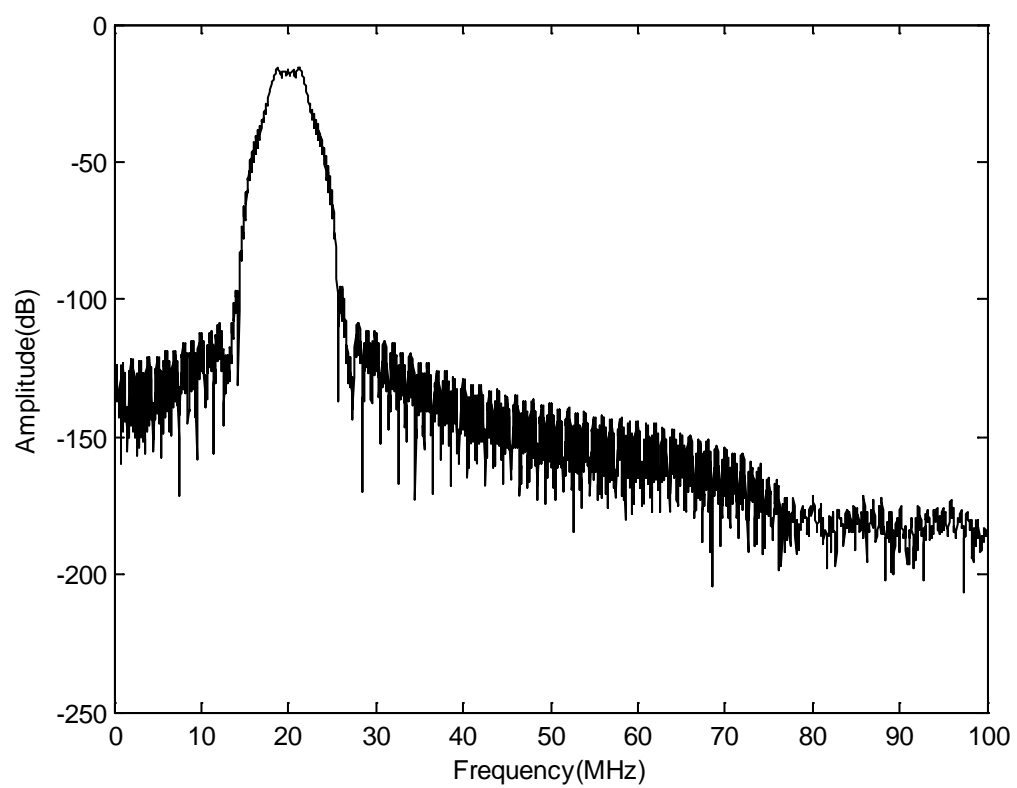

Figure 14. IF output signal spectrum.

The nonlinear parameters of the mixer and amplifier are set as shown in Table 7. According to the theoretical calculation, the suppression of the second-order harmonic component relative to the fundamental frequency in the mixer output signal should be 35 $\mathrm{dBm}$, and the suppression of the third-order harmonic component relative to the fundamental frequency should be $44 \mathrm{dBm}$. The output signal of mixer is shown in Figure 15. It can be seen that the simulation results are basically consistent with the theory.

Table 7. Nonlinear parameter index of transmitting channel.

\begin{tabular}{ccc}
\hline & Parameter & Value \\
\hline \multirow{3}{*}{ Mixer } & Third order truncation point & $27 \mathrm{dBm}$ \\
& Second order truncation point & $37 \mathrm{dBm}$ \\
& $1 \mathrm{~dB}$ compression point & $14 \mathrm{dBm}$ \\
\hline \multirow{3}{*}{ Amplifier } & Third order truncation point & $22 \mathrm{dBm}$ \\
& Second order truncation point & $30 \mathrm{dBm}$ \\
& $1 \mathrm{~dB}$ compression point & $17 \mathrm{dBm}$ \\
\hline
\end{tabular}

According to the theoretical calculation, the suppression of the third-order harmonic component relative to the fundamental frequency in the amplifier output signal should be $96 \mathrm{dBm}$. The output signal of amplifier is shown in Figure 16. It can be seen that the simulation results are basically consistent with the theory. 


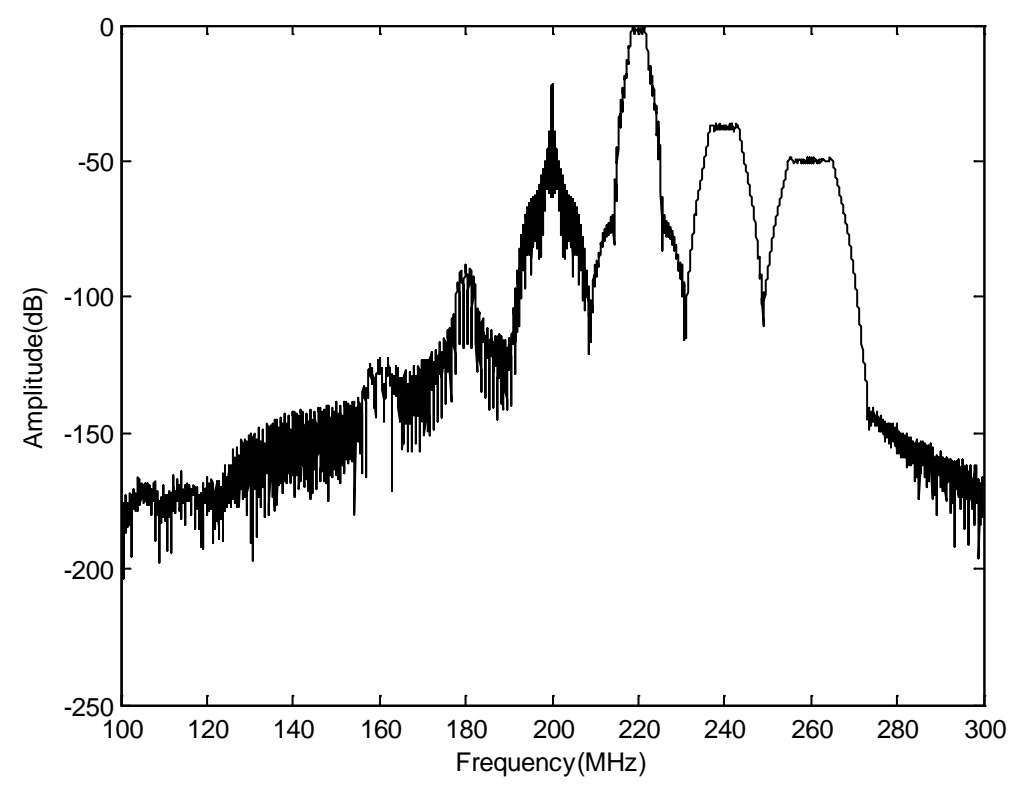

Figure 15. Frequency spectrum of mixer output signal.

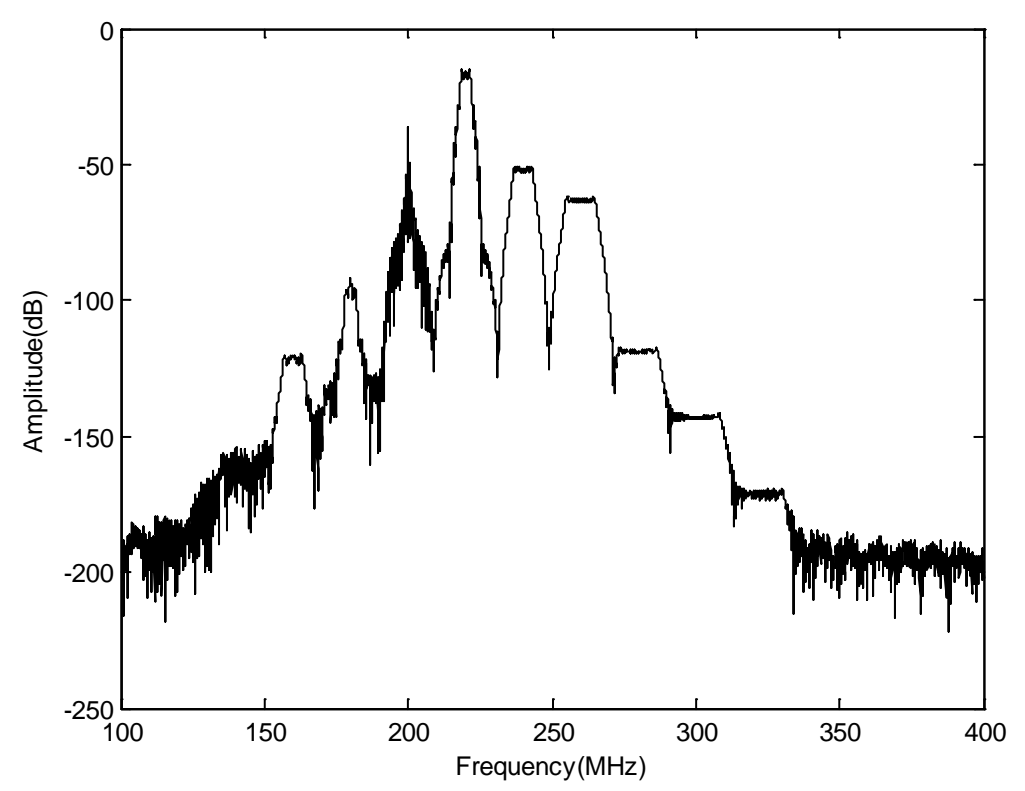

Figure 16. Frequency spectrum of amplifier output signal.

\subsection{Verification of Fingerprint Identification Effectiveness}

In this section, four typical radar emitter fingerprint recognition algorithms are selected to verify the effectiveness of the fingerprint characteristics identification of the data generated by the above model. The four algorithms use the front edge of pulse envelope [37], bispectral diagonal slice [19], Zero-Slice feature of ambiguity function [18] and inter pulse information parameters [38] as fingerprint features to identify radar emitter individuals.

Based on the fingerprint-level simulation model of typical radar emitter, five different individual radar emitters of the same type are simulated by changing the nonlinear design parameters of the model (such as $P_{i n-1 d B}, \mathrm{IIP}_{3}$ and $\mathrm{IIP}_{2}$ ), and 1000 sample signals are generated for each individual emitter under the same working parameters and modulation mode (all are LFM signals with starting frequency of $200 \mathrm{MHz}$, termination frequency of $220 \mathrm{MHz}$ and pulse width of 5us), the validity of fingerprint feature identifiability of simulation data is tested under different SNR conditions. As shown in Figure 17, from top 
to bottom and from left to right are the normalized fingerprint features extracted by five different individuals under three kinds of recognition algorithms [18,19,37]. It can be seen that the fingerprint features extracted by different individuals have subtle differences. The experimental results are shown in Table 8 . It is proved that the signal data generated by the simulation model contains effective fingerprint features, which can be reliable experimental data for the radar emitter individual identification algorithm.
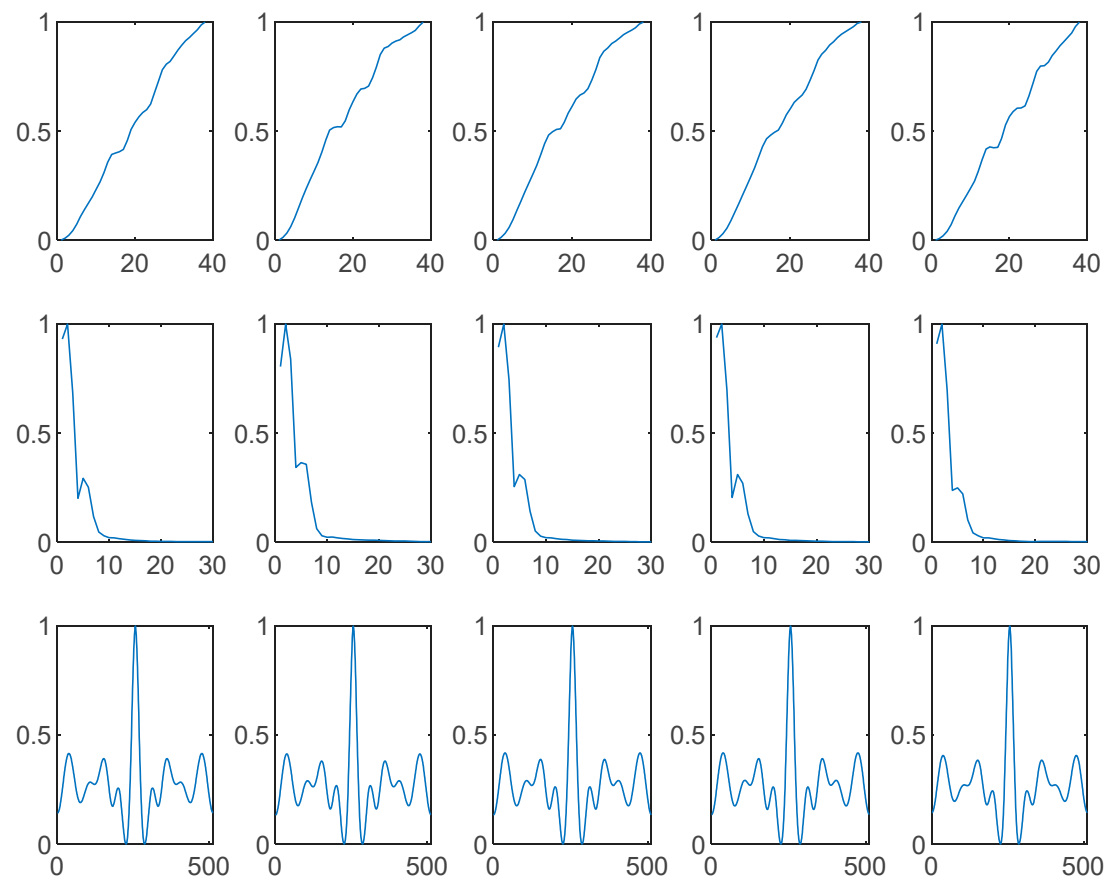

Figure 17. Comparison of normalized fingerprint characteristics of five individuals.

Table 8. Fingerprint feature identifiability test results of simulation model data.

\begin{tabular}{ccccc}
\hline Signal to Noise Ratio & Algorithm [37] & Algorithm [19] & Algorithm [18] & Algorithm [38] \\
\hline $30 \mathrm{~dB}$ & $92.2 \%$ & $89.9 \%$ & $99.8 \%$ & $100.0 \%$ \\
$20 \mathrm{~dB}$ & $67.8 \%$ & $67.4 \%$ & $87.8 \%$ & $90.1 \%$ \\
$10 \mathrm{~dB}$ & $37.2 \%$ & $37.0 \%$ & $56.0 \%$ & $35.0 \%$ \\
\hline
\end{tabular}

\section{Conclusions}

In this paper, we first investigate the structure of the typical radar emitter, complete the fingerprint-level radar emitter simulation model on the Systemvue simulation platform, and verify the nonlinear characteristics of the core module. Finally, we test the fingerprint feature identifiability on two kinds of typical fingerprint recognition algorithms, and the experimental results show that the data generated by the simulation model is effective-it can be reliable experimental data for radar emitter fingerprint identification. Moreover, the simulation model is different from the previous single component modeling method, which can only produce data under the condition of single modulation mode and working parameters. It can provide theoretical analysis and data support experimental conditions for fingerprint identification under various modulation modes and working parameters.

Author Contributions: Conceptualization, P.M., C.D. and W.R.; Methodology, P.M. and C.D.; Software, P.M.; Validation, P.M.; Formal Analysis, P.M.; Investigation, P.M.; Resources, G.X.; Data Curation, P.M.; Writing-Original Draft Preparation, P.M.; Writing-Review and Editing, C.D. and W.R.; Visualization, P.M.; Supervision, C.D.; Project Administration, G.X.; Funding Acquisition, G.X. All authors have read and agreed to the published version of the manuscript. 
Funding: This research received no external funding.

Data Availability Statement: The data presented in this study are all simulated by the model we proposed.

Acknowledgments: We express our thanks to the editors and anonymous reviewers for their valuable comments.

Conflicts of Interest: The authors declare no conflict of interest.

\section{References}

1. Talbot, K.I.; Duley, P.R.; Hyatt, M.H. Specific Emitter Identification and Verification. Technol. Rev. J. 2003, 113-133.

2. $\mathrm{Xu}, \mathrm{D}$. Research on Mechanism and Methodology of Specific Emitter Identification. Ph.D. Thesis, National University of Defense Technology, Changsha, China, April 2008.

3. He, M. Radar Countermeasure Information Processing, 1st ed.; Tsinghua University Press: Beijing, China, 2010.

4. Satija, U.; Trivedi, N.; Biswal, G.; Ramkumar, B. Specific emitter identification based on variational mode decomposition and spectral features in single hop and relaying scenarios. IEEE Trans. Inf. Forensics Secur. 2019, 14, 581-591. [CrossRef]

5. Guo, S.; White, R.E.; Low, M. A comparison study of radar emitter identification based on signal transients. In Proceedings of the 2018 IEEE Radar Conference, Oklahoma City, OK, USA, 23-27 April 2018.

6. Wu, L.; Zhao, Y.; Feng, M.; Abdalla, F.Y.; Ullah, H. Specific emitter identification using IMF-DNA with a joint feature selection algorithm. Electronics 2019, 8, 934. [CrossRef]

7. Ru, X.H.; Liu, Z.; Huang, Z.T.; Jiang, W.L. Evaluation of unintentional modulation for pulse compression signals based on spectrum asymmetry. IET Radar Sonar Navig. 2017, 11, 656-663. [CrossRef]

8. Wang, H.; Zhang, T.; Meng, F. Specific emitter identification based on time-frequency domain characteristic. J. Inf. Eng. Univ. 2018, 19, 23-29.

9. Zhang, J.; Wang, F.; Dobre, O.A.; Zhong, Z. Specific emitter identification via Hilbert-Huang transform in single-hop and relaying scenarios. IEEE Trans. Inf. Forensics Secur. 2016, 11, 1192-1205. [CrossRef]

10. Ren, D.F.; Zhang, T.; Jie, H.A.N.; Wang, H.H. Specific emitter identification based on ITD and texture analysis. J. Commun. 2017, 38, 160-168.

11. Huang, G.; Yuan, Y.; Wang, X.; Huang, Z. Specific emitter identification based on nonlinear dynamical characteristics. Can. J. Electr. Comput. Eng. 2016, 39, 34-41. [CrossRef]

12. Ye, W.; Peng, C. Recognition algorithm of emitter signals based on PCA+CNN. In Proceedings of the 2018 IEEE 3rd Advanced Information Technology, Electronic and Automation Control Conference, Chongqing, China, 12-14 October 2018; pp. 2410-2414.

13. Ding, L.; Wang, S.; Wang, F.; Zhang, W. Specific emitter identification via convolutional neural networks. IEEE Commun. Lett. 2018, 22, 2591-2594. [CrossRef]

14. Mcginthy, J.M.; Wong, L.J.; Michaels, A.J. Groundwork for neural network-based specific emitter identification authentication for IoT. IEEE Internet Things J. 2019, 6, 6429-6440. [CrossRef]

15. Wu, X.; Shi, Y.; Meng, W.; Ma, X.; Fang, N. Specific emitter identification for satellite communication using probabilistic neural networks. Int. J. Satell. Commun. Netw. 2019, 37, 283-291. [CrossRef]

16. Dudczyk, J. A method of feature selection in the aspect of specific identification of radar signals. Bull. Pol. Acad. Sci. Tech. Sci. 2017, 65, 113-119. [CrossRef]

17. Dudczyk, J.; Kawalec, A. Specific emitter identification based on graphical representation of the distribution of radar signal parameters. Bull. Pol. Acad. Sci. Tech. Sci. 2015, 63, 391-396. [CrossRef]

18. Wang, L. On Methods for Specific Radar Emitter Identification. Ph.D. Thesis, Xidian University, Xi'an, China, September 2011.

19. Ren, L. Research on Emitter Fingerprint Identification and Fine Feature Extraction. Master's Thesis, Harbin Engineering University, Harbin, China, March 2012.

20. Yu, Z. Study on Radar Emitter Signal Identification based on Intra-pulse Features. Ph.D. Thesis, Southwest Jiaotong University, Chengdu, China, January 2010.

21. Yu, Q. Individual identification of communication emitter based on permutation entropy fractal dimension feature extraction. $J$. Air Force Radar Acad. 2017, 31, 184-189.

22. Han, T. Research on the Techniques of Specific Emitter Identification for Pulse Signals. Ph.D. Thesis, National University of Defense Technology, Changsha, China, September 2013.

23. Ding, L.; Geng, F.; Chen, J. Principle of Radar, 3rd ed.; Electronics Industry Press: Beijing, China, 2014.

24. Guo, Y.; Lu, W. Design of Ground Transmitter System for Space Tracking and Communication, 1st ed.; National Defense Industry Press: Beijing, China, 1984.

25. Guo, Y. Frequency Stability of Modern Electronic Equipment, 1st ed.; China Astronautic Publishing House: Beijing, China, 1989.

26. Zhang, G. Research on Emitter Identification. Ph.D. Thesis, National University of Defense Technology, Changsha, China, March 2005.

27. Danev, B.; Capkun, S. Transient-based identification of wireless sensor nodes. In Proceedings of the 2009 International Conference on Information Processing in Sensor Networks, San Francisco, CA, USA, 13-16 April 2009; pp. 25-36. 
28. Yuan, Y.; Huang, Z.; Wu, H.; Wang, X. Specific emitter identification based on Hilbert-Huang transform-based time-frequency energy distribution features. IET Commun. 2014, 8, 2404-2412. [CrossRef]

29. Ye, H.; Liu, Z.; Jiang, W. A comparison of unintentional modulation on pulse features with the consideration of Doppler effect. J. Electron. Inf. Technol. 2012, 34, 118-123. [CrossRef]

30. Dudczyk, J.; Wnuk, M. The utilization of unintentional radiation for identification of the radiation sources. In Proceedings of the 34th European Microwave Conference, Amsterdam, The Netherlands, 12-14 October 2004.

31. Kawalec, A.; Rapacki, T.; Wnuczek, S.; Dudczyk, J.; Owczarek, R. Mixed Method Based on Intrapulse Data and Radiated Emission to Emitter Sources Recognition. In Proceedings of the International Conference on Microwaves, Krakow, Poland, 22-24 May 2006.

32. Nicholas, H.T.; Samueli, H. An analysis of the output spectrum of direct digital frequency synthesizers in the presence of phase accumulator truncation. In Proceedings of the 41st Annual Symposium on Frequency Control, Philadelphia, PA, USA, 27-29 May 1987; pp. 495-502.

33. Tranter, W.H.; Rappaport, T.S.; Kosbar, K.L.; Shanmugan, K.S. Principles of Communication Systems Simulation with Wireless Applications; Prentice Hall Professional Technical Reference: Upper Saddle River, NJ, USA, 2004; p. 7458.

34. Lin, Q.; Zhang, Z.; Guo, W. Analysis of Microwave Power Amplifier Nonlinear Distortion. J. Microw. 2004, 20 , 79-82.

35. Pozar, D.M. Microwave Engineering, 4th ed.; John Wiley \& Sons: New York, NY, USA, 2011.

36. Liu, M.W.; Doherty, J.F. Specific emitter identification using nonlinear device estimation. In Proceedings of the 2008 IEEE Sarnoff Symposium, Princeton, NJ, USA, 28-30 April 2008; pp. 1-5.

37. Yang, F. The Feature Extraction of Radar Source and Radar Individual Identification. Master's Thesis, Xidian University, Xi'an, China, May 2017.

38. Chen, J. Features Selection and Specific Emitter Identification of Radar. Master's Thesis, Xidian University, Xi'an, China, January 2013. 